\title{
Effectiveness of pneumococcal conjugate vaccine and rotavirus vaccine introduction into the South African public immunisation programme
}

\author{
S A Madhi, ${ }^{1-3}$ MB BCh, MMed, FCPaed, PhD; L Bamford, ${ }^{4} \mathrm{MB}$ ChB, BSocSci (Hons), FCPaed, DrPH; N Ngcobo ${ }^{4}$ MB BCh \\ ${ }^{1}$ National Institute for Communicable Diseases, National Health Laboratory Service, Sandringham, Johannesburg, South Africa \\ ${ }^{2}$ Medical Research Council Respiratory and Meningeal Pathogens Research Unit, University of the Witwatersrand, Johannesburg, South Africa \\ ${ }^{3}$ Department of Science and Technology/National Research Foundation: Vaccine Preventable Diseases, University of the Witwatersrand, \\ Johannesburg, South Africa \\ ${ }^{4}$ Child and Youth Health Directorate, National Department of Health, Pretoria, South Africa
}

Corresponding author: S A Madhi (shabirm@nicd.ac.za)

Immunisation has contributed greatly to the control of vaccine-preventable diseases and therefore to improvements in health and survival, especially among young children, and remains one of the most successful and cost-effective public health interventions. This remains true for many of the newer, more expensive vaccines. Vaccines against invasive pneumococcal disease and rotavirus infection were introduced into the South African Expanded Programme on Immunization in April 2009. This article describes the rationale for and process of the introduction of these two vaccines, pneumococcal conjugate vaccine and rotavirus vaccine. It also aims to evaluate the success of and challenges related to their introduction, in terms of both achieving universal coverage and improving survival and health in South African children.

S Afr Med J 2014;104(3 Suppl 1):228-234. DOI:10.7196/SAMJ.7597

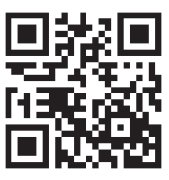

\section{Rationale for pneumococcal conjugate vaccine and rotavirus vaccine introduction into public immunisation programmes}

While progress has been made in preventing deaths due to some of the vaccine-preventable diseases (e.g. measles deaths reduced from 535000 in 2000 to 139000 by 2010), ${ }^{[1]}$ a greater proportion of vaccine-preventable deaths is now attributed to diseases for which there are under-utilised and/or newly developed vaccines, such as those against pneumococcal disease and rotavirus. Although these vaccines are widely used in developed and well-resourced countries, their use in middle- and lower-income countries has remained limited, mainly owing to the high costs of the vaccines.

Worldwide it was estimated that pneumococcal disease was responsible for 14 million severe cases and 830000 deaths in $2000,{ }^{[2]}$ and that there were 7 million severe cases of rotavirus diarrhoea and 453000 deaths in $2004 .{ }^{[3]}$ Significant declines in severe disease and/or mortality from these pathogens have been demonstrated in countries where the newer vaccines were introduced into public immunisation programmes. ${ }^{[4,5]}$ In the absence of targeted vaccination, rotavirus remains the most common cause of severe diarrhoea (associated with $28 \%$ of cases) and Streptococcus pneumoniae is still responsible for a high proportion (18.3\%) of cases of severe pneumonia. ${ }^{[6]}$

In 2007, the World Health Organization (WHO) recommended that countries with under-5 mortality rates of $>50 / 1000$ live births, and with a high prevalence of HIV or conditions that increase the risk of pneumococcal disease, should prioritise the introduction of the 7-valent pneumococcal conjugate vaccine (PCV-7) in their national immunisation programmes. ${ }^{[7]}$ Similar recommendations for rotavirus were subsequently issued. ${ }^{[8]}$
These global recommendations were premised in part by pivotal studies that had been undertaken in South Africa (SA). These included a study on a 9-valent PCV in which vaccine efficacy was established against vaccine-serotype invasive pneumococcal disease in HIV-uninfected (83\%) and HIV-infected children not on antiretroviral therapy (ART) (63\%), as well as against pneumonia in the first 2 years of life. ${ }^{[9]}$ Also, a vaccine efficacy study on rotavirus vaccine (RV) observed $73 \%$ protection against rotavirus-associated severe diarrhoeal disease in the SA setting, with its high prevalence of HIV infection. ${ }^{[10]}$ Although the efficacy study on RV was not specifically powered to evaluate for protection in HIV-infected children, the immunogenicity and safety of RV in this group had been established in a separate SA study. ${ }^{[1]]}$

\section{Introduction of PCV and RV into the SA public immunisation programme}

SA was the first country in Africa to introduce PCV and RV into its routine Expanded Programme on Immunization (EPI). PCV-7 was introduced in April 2009, followed by RV in August of the same year. The 13-valent pneumococcal conjugate vaccine (PCV-13), estimated to confer a further $20 \%$ protection against invasive pneumococcal disease (IPD), replaced PCV-7 in May 2011. ${ }^{[7]}$ The introduction of these vaccines into the SA EPI was particularly notable, this country having been classified as a middle-income country and therefore ineligible for external donor support from the Global Alliance for Vaccines and Immunization for procurement of these vaccines. Consequently in SA, where all healthcare for children is free of charge, government increased its budget allocation for the procurement of childhood vaccines from approximately R200 million to R1.25 billion per annum. Implementation of the new vaccines (which are packaged as prefilled single-dose syringes) also required further investment for doubling cold-chain capacity at both depot and 
facility level (personal communication - EPI, National Department of Health).

PCV and RV dosing schedule in SA

The EPI schedule, which includes RV and PCV, is available online (http://www.doh.gov.za). On the advice of the National Advisory Group for Immunization (NAGI), SA adopted a dosing schedule different to that recommended by the WHO at the time. ${ }^{[12]}$ In contrast to the WHO schedule, which recommended that PCV be given at 6,10 and 14 weeks, ${ }^{[7]}$ the $\mathrm{PCV}$ dosing schedule used in SA is a primary series at 6 and 14 weeks of age and a booster dose at 9 months of age, aimed at possibly extending the duration of protection against IPD in HIV-infected children, who at the time of implementation accounted for two-thirds of cases. ${ }^{[13]}$ This was decided upon to address specific observations of waning immunity among HIV-infected children noted in the SA PCV vaccine efficacy trial, ${ }^{[14]}$ and also to manage logistical issues with the simultaneous introduction of PCV and RV into the EPI. The immunogenicity of the implemented PCV dosing schedule has since been confirmed in SA children, including demonstration of higher antibody concentrations after the third dose at age 9 months compared with administration at age 14 weeks in the 6-, 10and 14-week dosing schedule recommended by the WHO. ${ }^{[15]}$

The rationale for RV being incorporated as a 6- and 14-week schedule was premised upon attempting to induce at least partial immunity as early as possible, i.e. the first dose at age 6 weeks rather than 10 weeks, while the decision to administer the second dose at 14 weeks was based on assisting with training in and the logistics of implementation of the concurrent introduction of PCV and RV into the EPI. The immunogenicity of RV using the 6- and 14-week schedule, including with the first dose being administered concurrently with the oral polio vaccine, has also been confirmed. ${ }^{[16]}$

\section{Coverage with PCV and RV}

The National Department of Health monitors the EPI programme performance using routine data submitted through the District Health Information System (DHIS). Coverage for a particular vaccine is calculated by dividing the number of doses that are reported to have been administered (predominantly in primary healthcare facilities) by the target population (based on mid-year population estimates published by Statistics South Africa and derived from census data).

The DHIS estimates for coverage with the third dose of PCV at 9 months of age increased from $10.4 \%$ in 2009 at the start of the PCV immunisation programme to $64.3 \%$ in 2010 and $99 \%$ in 2012 (unpublished data - DHIS, extracted May 2013) (Fig. 1). The $>100 \%$ coverage rates observed in provinces such as Gauteng

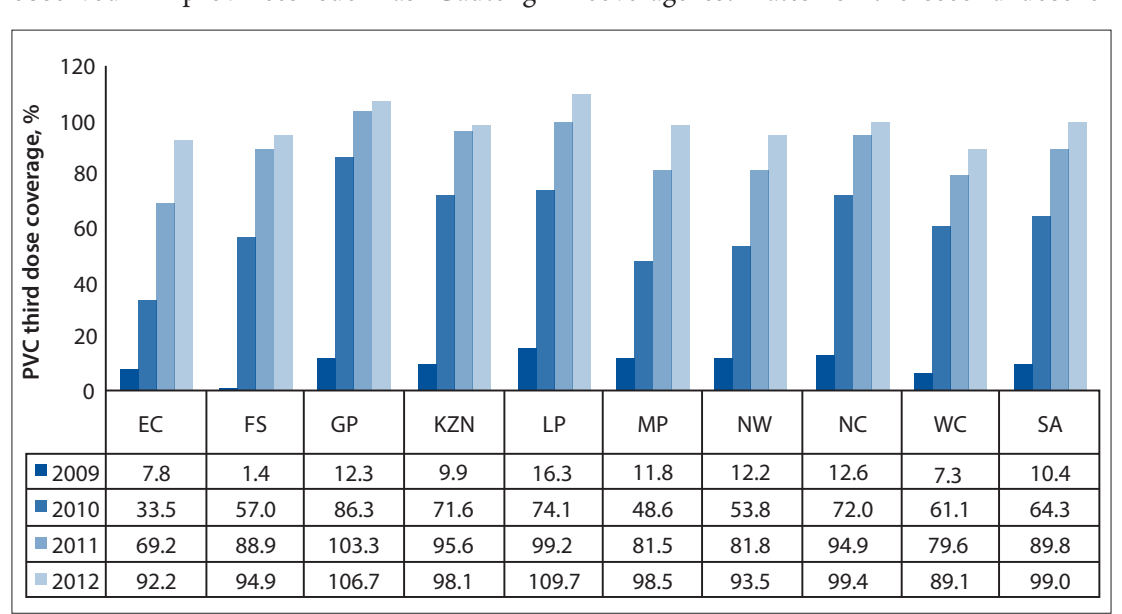

Fig. 1. District Health Information Systems estimates of coverage rates for the third dose of pneumococcal conjugate vaccine scheduled for administration at 9 months of age by province and nationally from 2009 to 2012. (PVC = pneumococcal conjugate vaccine; $E C=$ Eastern Cape; FS = Free State; $G P=$ Gauteng; $K Z N=$ KwaZulu-Natal; $L P=$ Limpopo; $M P=$ Mpumalanga; $N W=$ North West NC = Northern Cape; WC $=$ Western Cape; $S A=$ South Africa).

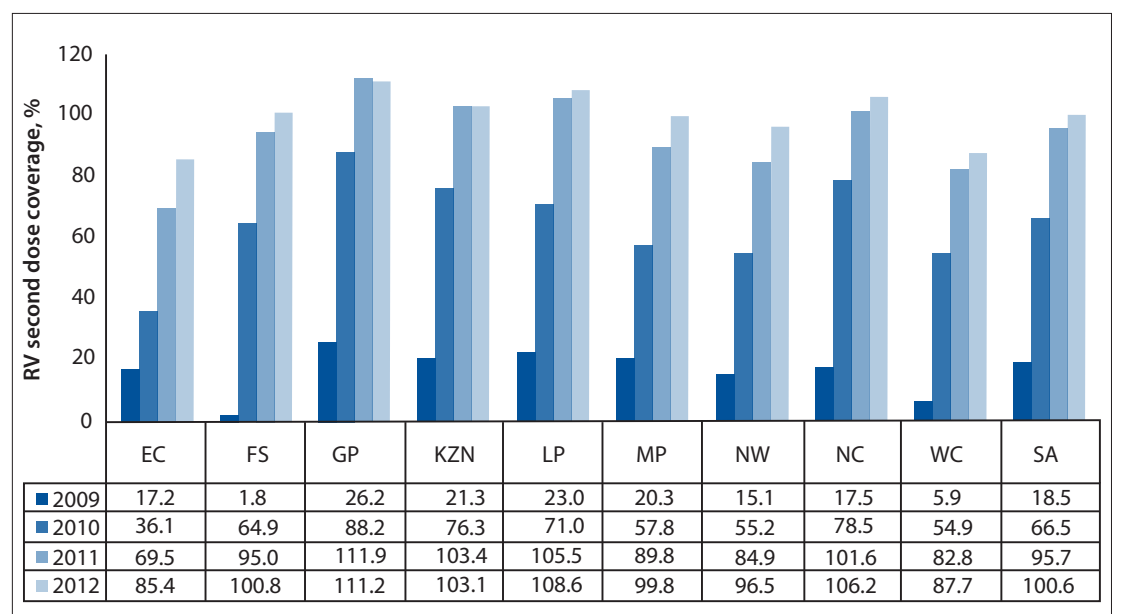

Fig. 2. District Health Information Systems estimates of coverage rates for the second dose of rotavirus vaccine administered at 14 weeks of age by province and nationally from 2009 to 2012. (RV = rotavirus vaccine; $E C=$ Eastern Cape; $F S=$ Free State; GP = Gauteng; KZN = KwaZulu-Natal; LP = Limpopo; $M P=$ Mpumalanga NW $=$ North West NC $=$ Northern Cape WC $=$ Western Cape SA = South Africa).

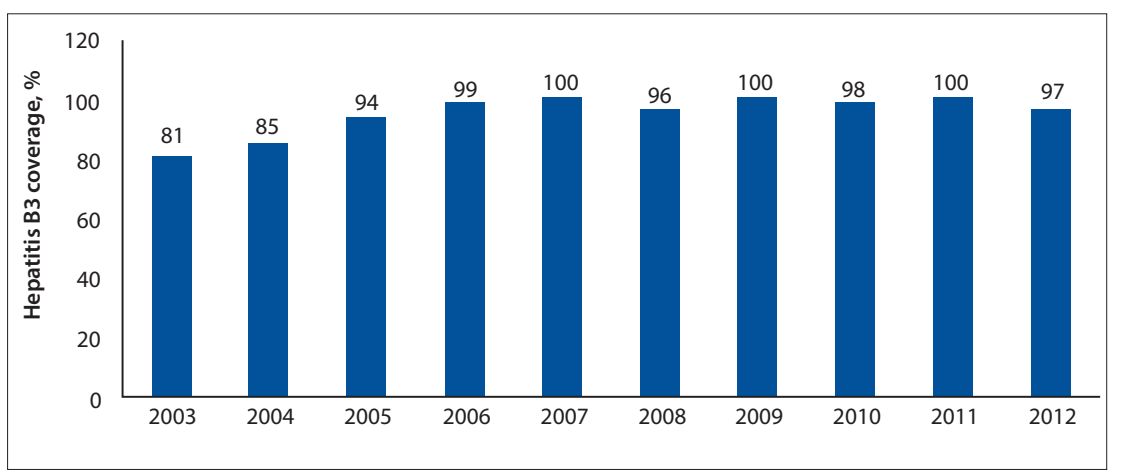

Fig. 3. District Health Information Systems estimates of coverage rates for the third dose of hepatitis $B$ vaccine administered at 14 weeks of age in South Africa from 2003 to 2012. 
RV administered at 14 weeks of age increased from $66.5 \%$ in 2010 to $100.6 \%$ in 2012 nationally (Fig. 2).

It should be noted that the DHIS reports high coverage for the traditional vaccines, e.g. coverage for the third dose of hepatitis $B$ vaccine has been reported to be between $96 \%$ and 100\% since 2005 (Fig. 3). These DHIS figures are not universally accepted as an accurate reflection of true coverage. For example, WHO/United Nations Children's Fund (UNICEF) estimates for PCV third-dose coverage were 72\% for 2011 and $81 \%$ for 2012 (compared with DHIS figures of $89 \%$ and $98 \%$, respectively), and $72 \%$ for 2011 and $78 \%$ for 2012 for the RV second dose (compared with DHIS figures of $78 \%$ and $100 \%$, respectively). ${ }^{[17]}$

The differences in coverage estimates for the two new vaccines are smaller than the differences in coverage estimates for more traditional vaccines (e.g. for 2012 the WHO/UNICEF estimated coverage of the third dose of DTP to be $68 \%$ compared with a DHIS figure of $93 \%$ ). This situation arises from the fact that WHO/UNICEF estimates of vaccine coverage are adjusted for differences in coverage rates between administrative reporting by government (i.e. DHIS figures in SA) and the actual coverage prevalence observed at the time of the last coverage survey undertaken in the country. In SA, the last representative population-based survey with immunisation coverage figures that were considered valid was undertaken in $1998{ }^{[18]}$ However, the WHO/UNICEF use coverage data from the 2003 Demographic and Health Survey ${ }^{[19]}$ which had limitations and did not produce reliable immunisation coverage figures. All subsequent WHO/UNICEF estimates have been adjusted downwards in line with the difference between administrative and survey estimates from 2003. A drawback of this methodology is that it is oblivious to any changes, e.g. strengthening or weakening of the EPI, which are likely to have occurred over more than a decade in a country like SA. Furthermore, WHO/UNICEF estimates are characterised by internal inconsistencies. For example, coverage estimates for the third doses of Haemophilus influenzae type $\mathrm{b}$ conjugate vaccine (HibCV) and diphtheria-tetanus (DTP) toxoid-whole cell pertussis (DTP combination vaccine) show considerable variation, despite the fact that the lyophilised HibCV is re-constituted with DTP in SA, so that the coverage for these vaccines should be identical.

Unfortunately, in the absence of a recent high-quality immunisation coverage survey in SA, vaccine coverage remains uncertain. However, both the DHIS data and the WHO/UNICEF data suggest that coverage of the new vaccines had reached levels comparable with those of the traditional vaccines 2 years after introduction.

\section{Effectiveness of PCV and RV in SA}

SA should have been well suited to measure the impact of the introduction of PCV and RV in reducing under-5 morbidity and mortality, owing to the structure of the healthcare system. In particular, although there is a parallel private sector, the majority $(>80 \%)$ of the population is cared for in the public health sector because of lack of private medical insurance coupled with the high cost of private care. Also, impediments to access to healthcare are addressed by free healthcare being provided to children $<6$ years of age, including hospitalisation and immunisation services. Unfortunately weaknesses in the vital registration systems, including delays in availability of updated mortality data, coupled with a limited healthcare information system, pose a challenge to accurately quantifying the cost-benefit and impact of the introduction of these new vaccines into the public healthcare system.

The usefulness of established and functional health information systems for demonstrating the impact of vaccines has been demonstrated in other middle-income countries such as Mexico, which has a similar gross domestic product and healthcare system to SA. The Mexican Ministry of Health electronic hospital registries show that RV reduced all-cause diarrhoea hospitalisation by $40 \%$, and all-cause diarrhoea mortality declined by $35 \%$ following RV introduction. ${ }^{[20,21]}$ Notably, such differences were evident in a diversity of socioeconomic settings in Mexico. ${ }^{[2]}$ Similarly, electronic databases were used in the USA to demonstrate a $36 \%$ reduction in all-cause pneumonia hospitalisation in children $<2$ years of age within $3-4$ years of PCV introduction, an effect that was sustained even a decade after PCV introduction. ${ }^{[23]}$ A number of other countries have also variably used available health data registries to measure the public health impact of PCV and RV on pneumonia and diarrhoea hospitalisation, respectively.

SA faces an additional challenge in understanding the impact of the introduction of PCV and RV in that this has taken place in parallel with changes in mother-to-child transmission (MTCT) of HIV programmes, as well as more effective management of HIV-infected children. Temporal trend data should therefore be interpreted in the context of these changes, which individually may impact on the incidence of pneumonia and diarrhoea as well as hospitalisation and mortality. In an effort to quantify the impact of the introduction of the two vaccines on child health and survival in SA, data from a number of sources were reviewed, and are summarised below.

\section{Case-control studies on effectiveness of PCV and RV}

Case-control studies have been undertaken to establish vaccine effectiveness for PCV and RV, to corroborate the results observed in the efficacy trials in SA. These have included studies to determine PCV vaccine effectiveness against pneumonia and IPD, as well as $\mathrm{RV}$ effectiveness against all-cause and rotavirus-associated diarrhoea hospitalisation. Using the case-control study design, a multi-centre study in SA reported that PCV vaccination was associated with a $69 \%(95 \%$ confidence interval $(\mathrm{CI}) 7$ - 90) reduced odds of hospitalisation for vaccine-serotype IPD and a $46 \%$ (95\% CI 0 - 71) lesser likelihood of hospitalisation for IPD due to any serotype in HIV-uninfected children older than 16 weeks of age. ${ }^{[24]}$ However, the study failed to confirm PCV effectiveness against vaccine-serotype or overall IPD in HIV-infected children, despite having access to ART. ${ }^{[24]}$ The reason for this remains unclear and has led to the NAGI recommending that HIV-infected children should receive an additional dose of PCV at 10 weeks of age (i.e. a $3+1$ dosing schedule). The immune responses following two doses of PCV given at 6 and 10 weeks of age were, however, similar in HIV-infected SA infants on ART compared with HIV-uninfected SA infants. ${ }^{[25]}$ Furthermore, provisional results of a case-control study also showed that PCV-immunised, HIV-uninfected children were 27\% (95\% CI 0 - 49) less likely than unimmunised children to be hospitalised for presumed bacterial pneumonia. ${ }^{[26]}$ A multi-centre, case-control study to evaluate the effectiveness of RV against rotavirus-associated diarrhoea hospitalisation is also nearing completion.

\section{Public health impact and surveillance data}

While case-control studies are useful for measuring the direct effectiveness of the vaccines, they are less informative in helping us understand the public health impact of the vaccines. The latter is best captured by examining temporal trends in disease in conjunction with introduction of various interventions, such as vaccines.

A limited number of ecological studies are underway in SA. These include a rotavirus surveillance study undertaken at sentinel hospital sites since 2009 that aims to measure the effectiveness of RV against diarrhoea hospitalisation. ${ }^{[27]}$ The results from this study reported that, compared with 2009 , there was a $60 \%$ (215 v. 534 cases) reduction in rotavirus diarrhoea hospitalisation in 2010 and a 64\% (190 v. 534 cases) 
reduction by 2011. Furthermore, there were also declines in hospitalisations for all-cause diarrhoea among infants, including a $34 \%$ (713/1 077) reduction in 2010 and a 31\% (742/1 077) reduction by 2011, compared with 2009. These data corroborate the findings of a review on the public health impact of RV in middle-income Latin American countries. ${ }^{[28}$ Observations from the review included that RV immunisation was associated with a 22 - $41 \%$ reduction in gastroenteritis-associated mortality, a 17 - 51\% reduction in all-cause gastroenteritis-associated hospitalisation, and a $59-81 \%$ reduction in rotavirus-specific gastroenteritis hospitalisation among children younger than 5 years of age. ${ }^{[28]}$

Additionally, an ecological study is underway to establish the effectiveness of PCV against IPD, for which there has been surveillance for more than a decade before vaccine introduction in SA. ${ }^{[29,30]}$ Provisional data up to the end of 2011, during which time 7-valent PCV was being used, showed a $79 \%$ reduction in vaccine-serotype IPD and a $28 \%$ decline in non-vaccine-serotype IPD in SA when comparing 2005 - 2008 with 2011. ${ }^{[30]}$ The greater decline in vaccineserotype IPD compared with non-vaccineserotype IPD suggests that while some of the decline may have been due to reduced MTCT of HIV and improved access to ART for $\mathrm{HIV}$-infected children, PCV immunisation also contributed. Other studies underway in SA include an ecological study in Soweto, Johannesburg, with a population of $\sim 1.25$ million people and an annual birth cohort of 29000 . This study aims to measure the effectiveness of PCV and RV immunisation on all-cause pneumonia and all-cause diarrhoea hospitalisation, which would provide more insight into the public health impact of immunisation with these vaccines, albeit in a limited geographical locality.

Although a national health usage registry has not been established in SA, some data on hospitalisation and in-hospital mortality are available. Included among these sources are the DHIS and Child Healthcare Problem Identification Programme (Child PIP). The DHIS collates data that are routinely submitted by public healthcare facilities. Data on the incidence of pneumonia and diarrhoea with dehydration in children $<5$ years of age are collected. Furthermore, data on in-hospital case fatality rates among children $<5$ years of age admitted to public hospitals are also collected. While the completeness and quality of data collected through the DHIS have improved substantially over the past years, trends should nevertheless be interpreted with care. Based on the DHIS data on pneumonia and diarrhoea in children $<5$ years of age for the period 2007 - 2012, the incidences for both diarrhoea and pneumonia have shown a downward trend (Table 1). This includes a steady decline in the incidence of diarrhoea with dehydration from 2008 to 2009 compared with later years, including a $44 \%$ reduction by 2012 . Furthermore, there was a decline in the incidence of pneumonia from $~ 96 / 1000$ in 2008 - 2009 to $69.3 / 1000$ by 2012 (a $28 \%$ decrease) (Table 1). There has been an $\sim 40 \%$ reduction in case fatality rates associated with diarrhoea and pneumonia (Table 1).

Another source of mortality data in public hospitals is available from Child PIP, a child mortality audit system that is increasingly being used in hospitals throughout SA. Data on the absolute number of under- 5 deaths from acute diarrhoea and pneumonia, and the proportions of all deaths with which they are associated, from 41 hospitals that have consistently submitted data from 2008 to 2012 are shown in Table 2. During this period, the number of deaths from diarrhoea has shown a steady decline, and was 56\% lower in $2012(N=244)$ than in $2008(N=556)$ (Table 2). Similarly, there has been a steady decline in deaths from pneumonia, which decreased by $53 \%$ between $2008(N=692)$ and $2012(N=326)$. The proportions of all under-5 deaths attributed to diarrhoea (19.4\% in 2008 and $16.2 \%$ in 2012 ) and pneumonia $(24.2 \%$ in 2008 and $21.6 \%$ in 2010 - 2012), while also showing a downward decline, nevertheless highlight the continuing contribution of these syndromes to under-5 mortality in SA (unpublished data, Medical Research Council, Child PIP, extracted 5 August). Analysis of a smaller number of hospitals $(N=18)$ that have submitted complete data

Table 1. District Health Information Systems data on diarrhoea and pneumonia in children $<5$ years of age at public health facilities, $2007-2012$

\begin{tabular}{lllllll}
\hline & 2007 & 2008 & 2009 & 2010 & 2011 & 2012 \\
\hline $\begin{array}{l}\text { Diarrhoea with dehydration incidence, } \\
\text { per } 1000 \text { children }<5 \text { years of age }\end{array}$ & 19.8 & 22.6 & 22.1 & 17.6 & 15.2 & 12.3 \\
$\begin{array}{l}\text { Pneumonia incidence, } \\
\text { per } 1000 \text { children }<5 \text { years of age }\end{array}$ & 87.4 & 92.8 & 100.0 & 82.7 & 83.2 & 69.3 \\
$\begin{array}{l}\text { Diarrhoea case fatality rate, } \\
\text { \% of children }<5 \text { years of age admitted to hospital }\end{array}$ & 8.4 & 8.8 & 7.3 & 7.0 & 5.2 & 4.2 \\
$\begin{array}{l}\text { Pneumonia case fatality rate, } \\
\% \text { of children }<5 \text { years of age admitted to hospital }\end{array}$ & 8.4 & 9.3 & 6.5 & 6.3 & 4.3 & 4.1
\end{tabular}

Table 2. Deaths of children $<5$ years of age from acute diarrhoea and pneumonia at hospitals with 5 years of complete data $(N=41), 2008-2012$

\begin{tabular}{llll}
\hline & & \multicolumn{2}{c}{ Deaths from, $\boldsymbol{n}(\%$ of $\boldsymbol{N})$} \\
\cline { 3 - 4 } 2008 & Total deaths, $\boldsymbol{N}$ & $556(19.4)$ & $692(24.2)$ \\
2009 & 2863 & $455(18.1)$ & $603(24.0)$ \\
2010 & 2511 & $392(17.4)$ & $487(21.6)$ \\
2011 & 2251 & $294(17.1)$ & $370(21.6)$ \\
2012 & 1716 & $244(16.2)$ & $326(21.6)$
\end{tabular}

Table 3. Deaths of children $<5$ years of age from acute diarrhoea and pneumonia at hospitals with 6 years of complete data $(N=18), 2007-2012$

\begin{tabular}{llll}
\hline & & \multicolumn{2}{c}{ Deaths from, $\boldsymbol{n}(\%$ of $\boldsymbol{N})$} \\
\cline { 3 - 4 } 2007 & Total deaths, $\boldsymbol{N}$ & $189(13.3)$ & $259(18.2)$ \\
2008 & 1426 & $248(17.3)$ & $274(19.1)$ \\
2009 & 1436 & $204(17.3)$ & $245(20.8)$ \\
2010 & 1177 & $165(16.5)$ & $152(15.2)$ \\
2011 & 1000 & $123(16.4)$ & $145(19.4)$ \\
2012 & 748 & $91(15.3)$ & $108(18.2)$
\end{tabular}


for the period 2007 - 2012 showed an increase in the number of deaths from diarrhoea and pneumonia between 2007 and 2008, followed by declines (Table 3 ).

Although data on the HIV status of children who die are collected during the Child PIP mortality audit process, the incompleteness of data makes interpretation thereof difficult. Data on the HIV-infection status of children who died from pneumonia (from the 18 hospitals with complete data) are shown in Table 4. Despite the increase from 2007 to 2012 in the percentage of children in whom HIV infection status was established at the time of their death, there has been a decline in the number of deaths involving HIV-infected children (from 80 in 2007 to 24 in 2012, a 70\% reduction). In contrast, the number of deaths of children who were confirmed as being HIV-uninfected has remained steadier over this time, albeit with an increase in the number in whom HIV testing was undertaken. This would therefore also suggest a decline in deaths among HIVuninfected children between 2007 and 2012 .

Table 5 shows the number of children $<5$ years of age from 18 hospitals whose deaths were attributed to diarrhoea (data from Child PIP). Similar to all-cause deaths, an increasing proportion of children who died of diarrhoea were tested for HIV infection in 2012 compared with earlier years; nevertheless, there was a trend towards a decline in diarrhoea deaths associated with HIV infection and among HIVuninfected children (Table 5). Importantly, the Child PIP database is not population denominator centred, and hence is unable to provide incidence trend data at a population level. Also, as the reporting is based on summary data for the hospitals instead of individual patient data, it is not amenable to being used to determine issues such as HIVstratified trends in incidence of diseases to help distinguish between the relative contribution of PCV and RV compared with changes in HIV management in reducing under-5 childhood mortality in SA.

\section{Child mortality estimates}

A significant decline (30\%) in under-5 mortality has been observed in SA between 2006 and 2011. The United Nations estimates that under5 mortality declined from 75.8/1 000 live births in 2006 to $46.7 / 1000$ live births in $2011,{ }^{[32]}$ and in addition there has been an estimated $48 \%$ reduction in diarrhoea-associated mortality and a $19 \%$ reduction in pneumonia mortality. ${ }^{[32]} \mathrm{A}$ major factor contributing to this decline in under- 5 mortality has been a $47 \%$ reduction in deaths attributed to HIV/AIDS and related complications. ${ }^{[32]}$ This accomplishment has been driven by improved management of HIV-infected children with ART since 2006, as well as improved coverage with a more aggressive ART regimen for pregnant women aimed at reducing MTCT of HIV. The rate of MTCT of HIV has declined from 9.6\% in 2008, when mainly nevirapine was used for the prevention of MTCT, to approximately $2.8 \%$ with more aggressive ART regimens having been implemented ${ }^{[33]}$ The model used for estimating the causes of under-5 mortality attributes all deaths in HIV-infected children to AIDS, although the major direct causes of death in these children are similar to those in the general population, i.e. pneumonia and diarrhoea. The risks of morbidity and mortality from common childhood illnesses such as pneumonia and diarrhoea are, however, much increased in HIV-infected children. Prior to the widespread management of HIV-infected children with ART, the risks of hospitalisation for pneumonia and diarrhoea were 9-fold and 5.4fold greater, respectively, among HIV-infected than HIV-uninfected SA children ${ }^{[3,3,35]}$ Also, HIV-infected children in SA had a 40-fold greater risk of hospitalisation for invasive pneumococcal disease and an estimated 2.3-fold increased risk of hospitalisation for rotavirus diarrhoeal disease than HIV-uninfected children ${ }^{[35,36]}$ Although improved access to ART has mitigated some of this risk, even in the era of ART HIV-infected children remain at a 20 -fold greater risk of developing invasive pneumococcal disease $^{[37]}$ Furthermore, even when ART was initiated early in HIV-

Table 4. HIV status of children $<5$ years of age who died from pneumonia at hospitals with 6 years of complete data $(N=18), 2007-2012$

\begin{tabular}{llllll}
\hline & HIV-negative & HIV-infected & \multicolumn{1}{l}{$\begin{array}{l}\text { HIV-exposed } \\
\boldsymbol{n}(\%)\end{array}$} & $\begin{array}{l}\text { Unknown } \\
\boldsymbol{n}(\%)\end{array}$ & $\begin{array}{l}\text { Total } \\
\boldsymbol{N}\end{array}$ \\
\hline 2007 & $30(11.6)$ & $80(30.9)$ & $75(29.0)$ & $74(28.6)$ & 259 \\
2008 & $60(21.9)$ & $70(25.5)$ & $76(27.7)$ & $68(24.8)$ & 274 \\
2009 & $54(22.0)$ & $49(20.0)$ & $87(35.5)$ & $55(22.4)$ & 245 \\
2010 & $32(21.1)$ & $46(30.3)$ & $49(32.2)$ & $25(16.4)$ & 152 \\
2011 & $53(36.6)$ & $39(26.9)$ & $28(19.3)$ & $25(17.2)$ & 145 \\
2012 & $45(41.7)$ & $24(22.2)$ & $23(21.3)$ & $16(14.8)$ & 108 \\
Total, $N$ & $274(23.2)$ & $308(26.0)$ & $338(28.6)$ & $263(22.2)$ & 1183 \\
${ }^{*}$ HIV-exposed with unknown HIV status. & & & & &
\end{tabular}

Table 5. HIV status of children $<5$ years of age who died from acute diarrhoea at hospitals with 6 years of complete data $(N=18), 2007$ - 2012

\begin{tabular}{|c|c|c|c|c|c|}
\hline & $\begin{array}{l}\text { HIV-negative } \\
n(\%)\end{array}$ & $\begin{array}{l}\text { HIV-infected } \\
n(\%)\end{array}$ & $\begin{array}{l}\text { HIV-exposed } \\
n(\%)\end{array}$ & $\begin{array}{l}\text { Unknown } \\
n(\%)\end{array}$ & $\begin{array}{l}\text { Total } \\
N\end{array}$ \\
\hline 2007 & $25(13.2)$ & $42(22.2)$ & $48(25.4)$ & $74(39.2)$ & 189 \\
\hline 2008 & $30(12.1)$ & $72(29.0)$ & $68(27.4)$ & $78(31.5)$ & 248 \\
\hline 2009 & $46(22.5)$ & $51(25.0)$ & $49(24.0)$ & $58(28.4)$ & 204 \\
\hline 2010 & $41(24.8)$ & $46(27.9)$ & $51(30.9)$ & $27(16.4)$ & 165 \\
\hline 2011 & $32(26.0)$ & 35 (28.5) & $32(26.0)$ & $24(19.5)$ & 123 \\
\hline 2012 & $31(34.1)$ & 29 (31.9) & $18(19.8)$ & $13(14.3)$ & 91 \\
\hline Total, $N$ & $205(20.1)$ & $275(27.0)$ & $266(26.1)$ & 274 (26.9) & 1020 \\
\hline
\end{tabular}




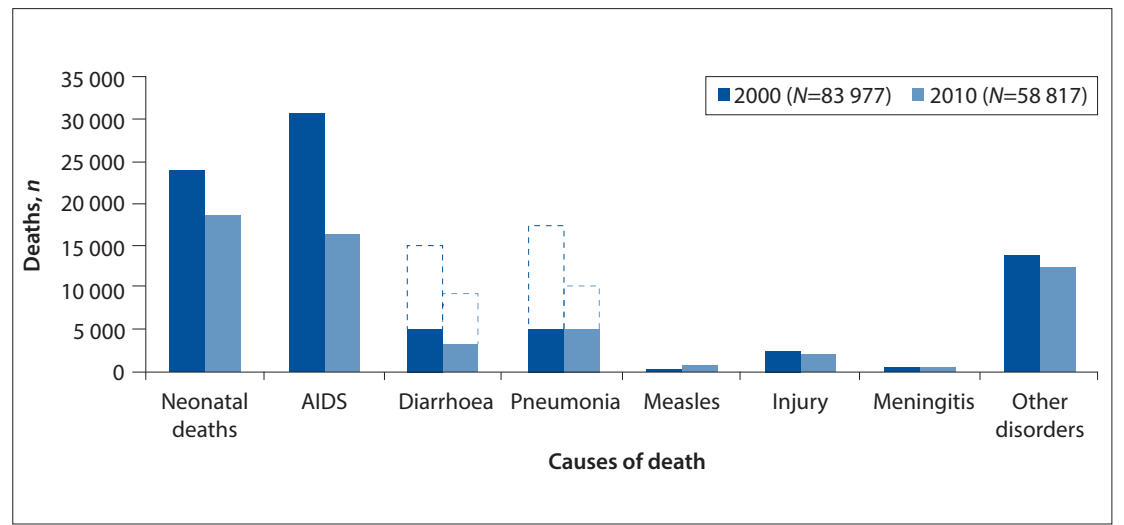

Fig. 4. Causes of under-5 childhood mortality in South African children in 2000 and 2010 (dashed bars extrapolate deaths due to pneumonia and diarrhoea in HIV-infected children who died). Figure derived from estimates by Liu et al, ${ }^{[32]}$ and imputed HIV/AIDS associated diarrhoea and pneumonia mortality based on a study by Violari et al.. ${ }^{[38]}$

infected infants, pneumonia and diarrhoea were individually identified as the causes of death in one-third of HIV-infected children. ${ }^{[38]}$

Extrapolating from the direct causes of death in SA children with HIV/AIDS, the imputed proportions of all under- 5 mortality that could be attributed to pneumonia and diarrhoea in 2010 were $~ 19 \%(N \approx 11000)$ and $\sim 15 \%$ ( $N \approx 9000$ ), respectively (Fig. 4). While further reduction in vertical transmission and improved ART management of HIV-infected children remain the mainstay of reducing HIV/ AIDS-associated morbidity and mortality, additional complementary interventions such as immunisation with new and under-utilised existing vaccines would contribute further towards reducing morbidity and mortality in this high-risk group.

\section{Conclusion}

SA has demonstrated a clear commitment to reducing child mortality and to ensuring universal access to life-saving vaccines by being at the forefront in introducing new vaccines such as PCV and RV into its childhood EPI. While exact coverage levels remain contested, coverage with the new vaccines has been scaled up rapidly to levels comparable to those of traditional vaccines.

The uncertainty around immunisation coverage remains a serious challenge, and in the absence of a recent high-quality survey in SA, vaccine coverage remains uncertain. This limits government's ability to accurately monitor and improve EPI performance. Furthermore, the WHO and UNICEF will not change their estimates of SA's coverage figures until a valid coverage survey is conducted, as they rely on recent coverage surveys to produce country coverage estimates and will not use only administrative figures reported by a country.
The effects of these new vaccines on under- 5 childhood morbidity and mortality in SA are challenging to measure with existing data sources. Overall child mortality rates have declined substantially in the past 5 years, while data from a number of sources show substantial declines in the incidence of diarrhoea with dehydration and pneumonia, as well as in hospitalisations and deaths from diarrhoea and pneumonia. However, since pneumonia and diarrhoea are also leading causes of death in HIV-infected children, the relative impact of improvements in HIV prevention and management is difficult to disentangle from the decline in pneumonia and diarrhoea morbidity and mortality due to the introduction of the vaccines. The reduction in diarrhoea mortality may at least in part be related to improved access to tapped potable water, which has been associated with significant global reductions in deaths from diarrhoea, and specifically diarrhoea caused by bacteria. ${ }^{[39]}$

As HIV-associated child mortality declines, further child survival gains will depend increasingly on preventing deaths among HIV-uninfected children. The reductions in hospitalisation of HIV-uninfected infants demonstrated in the case-control studies suggest that these vaccines will play an increasing role in preventing child morbidity and mortality in the future.

Further challenges in addressing the effectiveness of PCV and RV immunisation in SA relate to the lack of electronic hospital registries, which would make measurement of the impact of PCV and RV on mortality and morbidity much simpler.

It is strongly recommended that a highquality immunisation coverage survey be conducted in every district and that an electronic hospital registry be implemented in SA. This would assist in better understanding of whether the substantial investment in vaccine procurement is translating into a benefit, particularly in impoverished districts and communities.

Conflict of interest. SAM has received grant support from GlaxoSmithKline (GSK) and Pfizer (Wyeth) for studies related to PCV and RV. SAM also received honoraria from GSK and Pfizer and served as a temporary advisor to Pfizer and GSK.

1. Simons E, Ferrari M, Fricks J, et al. Assessment of the 2010 global measles mortality reduction goal: Results from a model of surveillance data. Lancet 2012:379(9832):2173-2178. [http:// of surveillance data. Lancet 2012;379(9832)
dx.doi.org/10.1016/S0140-6736(12)60522-4] 2. Parashar UD, Burton A, Lanata C, et al. Global mortality associated with rotavirus disease among children in 2004. J Infect

Dis 2009;200(Suppl 1):S9-S15. [http:///dx.doi.org/10.1086/605025]
O'Brien KL, Wolfson LJ, Watt JP, et al. Burden of disease caused . O'Brien KL, Wolfson LJ, Watt JP, et al. Burden of disease caused
by Streptococcus pneumoniae in children younger than 5 years: Global estimates. Lancet 2009;374(9693):893-902. [http:// dx.doi.org/10.1016/S0140-6736(09)61204-6]

4. Tate JE, Patel MM, Steele AD, et al. Global impact of rotavirus vaccines. Expert Rev Vaccines 2010;9(4):395-407. [http://dx.doi. org/10.1586/erv.10.17]

5. Walker CL, Rudan I, Liu L, et al. Global burden of childhood pneumonia and diarrhoea. Lancet 2013;381(9875):1405-1416. [http://dx.doi.org/10.1016/S0140-6736(13)60222-6]

6. Progress in introduction of pneumococcal conjugate vaccine - worldwide, 2000-2012. MMWR Morb Mortal Wkly Rep 2013;62(16):308-311.

7. World Health Organization. Pneumococcal conjugate vaccine for childhood immunization - WHO position paper. Wkly Epidemiol Rec 2007;82(12):93-104.

8. World Health Organization. Rotavirus vaccine: An update. Wkly Epidemiol Rec 2009;84(51-52):533-538.

9. Klugman KP, Madhi SA, Huebner RE, Kohberger R, Mbelle N, Pierce N. A trial of a 9-valent pneumococcal conjugate vaccine in children with and those without HIV infection. N Engl J Med 2003;349(14):1341-1348. [http://dx.doi.org/10.1056/ NEJMoa035060]

10. Madhi SA, Cunliffe NA, Steele D, et al. Effect of human rotavirus vaccine on severe diarrhoea in African infants. N Engl J Med 2010;362(4):289-298. [http://dx.doi.org/10.1056/NEJMoa0904797]

11. Steele AD, Madhi SA, Louw CE, et al. Safety, reactogenicity, and immunogenicity of human rotavirus vaccine RIX4414 in human immunodeficiency virus-positive infants in South in human immunodeficiency virus-positive infants in South
Africa. Pediatr Infect Dis J 2011;30(2):125-130. [http://dx.doi. Africa. Pediatr Infect Dis J 2011;30(2):
org/10.1097/INF.0b013e3181f42db9]

12. Recommendations for the prevention of Streptococcus Recommendations for the prevention of Streptococcus
pneumoniae infections in infants and children: Use of 13-valent pneumoniae infections in infants and children: Use of 13-valent
pneumococcal conjugate vaccine (PCV13) and pneumococcal polysaccharide vaccine (PPSV23). Pediatrics 2010;126(1):186190. [http://dx.doi.org/10.1542/peds.2010-1280]

13. Nunes MC, von Gottberg A, de Gouveia L, et al. The impact of antiretroviral treatment on the burden of invasive pneumococcal disease in South African children: A time series analysis. AIDS 2011;25(4):453-462. [http://dx.doi.org/10.1097/ QAD.0b013e328341b7f1]

14. Madhi SA, Adrian P, Kuwanda L, et al. Long-term immunogenicity and efficacy of a 9 -valent conjugate pneumococcal vaccine in human immunodeficient virus infected and non-infected children in the absence of a booster dose of vaccine. Vaccine 2007;25(13):24512457. [http://dx.doi.org/10.1016/j.vaccine.2006.09.019]

15. Jones SA, Groome M, Koen A, et al. Immunogenicity of sevenJones SA, Groome M, Koen A, et al. Immunogenicity of seven-
valent pneumococcal conjugate vaccine administered at 6,14 and 40 valent pneumococcal conjugate vaccine administered at 6,14 and 40
weeks of age in South African infants. PloS One 2013;8(8):e72794. weeks of age in South African infants. PloS One 2013:
[http://dx.doi.org/10.1371/journal.pone.0072794]

16. Groome MJ, Moon S-S, Velasquez D, et al. Effect of breastfeeding on immunogenicity of oral live-attenuated human rotavirus vaccine: A randomized trial in HIV-uninfected infants in Soweto, South Africa. Bull World Health Org 2014 (In press).

7. UNICEF and WHO, Immunization Summary. A Statistical reference containing data through 2011, 2013 edition. UNICEF, New York. 2013. http://www.unicef.org/videoaudio/PDFs/ENImmSumm-2013.pdf (accessed 24 September 2013).

18. Medical Research Council and Department of Health. SA Memographic \& Health Survey 1998. Pretoria. Department Demographic \& Health Survey 1998. Pretoria: Department of Health, 1998. http://www.mrc.ac.za/bod/demographicsurvey.
htm (accessed 24 September 2013).

. Department South Africa Demographic and Health Survey 2003. Pretoria: Department of Health, 2007. http://www.mrc.ac.za/bod/sadhs.
htm (accessed 24 September 2013).

20. Richardson V, Hernandez-Pichardo J, Quintanar-Solares M, et al. Effect of rotavirus vaccination on death from childhood diarrhoea in Mexico. N Engl J Med 2010;362(4):299-305. [http:// dx.doi.org/10.1056/NEJMoa0905211] 
21. Quintanar-Solares M, Yen C, Richardson V, Esparza-Aguilar M, Parashar UD, Patel MM. Impact of rotavirus vaccination on diarrhoea-related hospitalizations among children $<5$ years of age in Mexico. Pediatr Infect Dis J 2011;30(1 Suppl):S11-S15. [http://dx.doi.org/10.1097/INF.0b013e3181fefb32]

22. Gastanaduy PA, Sanchez-Uribe E, Esparza-Aguilar M, et al. Effect of rotavirus vaccine on diarrhoea mortality in different socioeconomic regions of Mexico. Pediatrics 2013;131(4):el115-el120. [http://
mation dx.doi.org/10.1542/peds.2012-2797]

23. Griffin MR, Zhu Y, Moore MR, Whitney CG, Grijalva CG. U.S. hospitalizations for pneumonia after a decade of pneumococcal vaccination. N Engl J Med 2013;369(2):155-163. [http://dx.doi.org/10.1056/ NEJMoa 1209165]

24. Cohen C, von Mollendorf C, Naidoo N, et al. Effectiveness of seven-valent pneumococcal conjugate vaccine against invasive pneumococcal disease in South Africa: A matched case-control study. Presented at the 8th International Symposium on Pneumococci and Pneumococcal Diseases, Iguaçu Falls, Brazil, 11-15 March 2012. Abstract 226.

25. Madhi SA, Izu A, Violari A, et al. Immunogenicity following the first and second doses of 7-valent pneumococcal conjugate vaccine in HIV-infected and -uninfected infants. Vaccine 2013;31(5):777783. [http://dx.doi.org/10.1016/j.vaccine.2012.11.076]

26. Madhi SA, Groome M, Zar H, et al. Effectiveness of pneumococcal conjugate vaccine against presumed bacterial pneumonia in South African HIV-uninfected children: A case control study. Presented at the bacterial pneumonia in South African HIV-uninfected children: A case control study. Presented at the
8th International Symposium on Pneumococci and Pneumococcal Diseases, Iguaçu Falls, Brazil, 11-15 March 2012. Abstract 304

27. Msimang VM, Page N, Groome MJ, et al. Impact of rotavirus vaccine on childhood diarrhoeal hospitalization following introduction into the South African public immunization program. Pediatr Infect Dis J 2013;32(12):1359-1364. [http://dx.doi.org/10.1097/INF.0b013e3182a72fc0]

28. Desai R, Oliveira LH, Parashar UD, Lopman B, Tate JE, Patel MM. Reduction in morbidity and mortality from childhood diarrhoeal disease after species A rotavirus vaccine introduction in Latin America - a review. Mem Instit Oswaldo Cruz 2011;106(8):907-911.

29. Von Gottberg A, Cohen C, de Gouveia L, et al. Epidemiology of invasive pneumococcal disease in the pre-conjugate vaccine era: South Africa, 2003-2008. Vaccine 2013;31(38):4200-4208. [http://dx.doi. org/10.1016/.vaccine.2013.04.077
30. Von Gottberg A, Cohen C, de Gouveia L, Quan V, et al. Trends in invasive pneumococcal disease after 7-valent pneumococcal conjugate vaccine introduction in South Africa, 2005-2011. Presented at the 8th International Symposium on Pneumococci and Pneumococcal Diseases, Iguaçu Falls, Brazil, 11-15 March 2012. Abstract 272 .

31. UNICEF, World Health Organization, World Bank, United Nations. Levels and trends in child mortality: Report 2012. Estimates developed by the UN Inter-agency group for Child Mortality Estimation. New York: UNICEF, 2012. http://www.unicef.org/videoaudio/PDFs/UNICEF_2012 child_mortality_for_web_0904.pdf (accessed 20 June 2013).

32. Liu L, Johnson HL, Cousens S, et al. Global, regional, and national causes of child mortality: An updated systematic analysis for 2010 with time trends since 2000. Lancet 2012;379(9832):2151-2161. [http://dx.doi.org/10.1016/S0140-6736(12)60560-1]

33. Barron P, Pillay Y, Doherty T, et al. Eliminating mother-to-child HIV transmission in South Africa. Bull World Health Organ 2013;91(1):70-74. [http://dx.doi.org/10.2471/BLT.12.106807]

34. Madhi SA, Kuwanda L, Cutland C, Klugman KP. The impact of a 9-valent pneumococcal conjugate vaccine on the public health burden of pneumonia in HIV-infected and -uninfected children. Clin Infect Dis 2005;40(10):1511-1518. [http://dx.doi.org/10.1086/429828]

35. Groome MJ, Madhi SA. Five-year cohort study on the burden of hospitalisation for acute diarrhoeal disease in African HIV-infected and HIV-uninfected children: Potential benefits of rotavirus vaccine. Vaccine 2012;30(Suppl 1):A173-A178. [http://dx.doi.org/10.1016/j.vaccine.2011.08.004]

36. Madhi SA, Petersen K, Madhi A, Wasas A, Klugman KP. Impact of human immunodeficiency virus type 1 on the disease spectrum of Streptococcus pneumoniae in South African children. Pediatr Infect type 1 on the disease spectrum of Streptococcus pneumoniae in South African children. P
Dis J 2000;19(12):1141-1147. [http://dx.doi.org/10.1097/00006454-200012000-00004]

Dis J 2000;19(12):1141-1147. [http://dx.doi.org/10.1097/00006454-200012000-00004]
37. Nunes MC, von Gottberg A, de Gouveia L, et al. The impact of antiretroviral treatment on the 37. Nunes MC, von Gottberg A, de Gouveia L, et al. The impact of antiretroviral treatment on the
burden of invasive pneumococcal disease in South African children: A time series analysis. AIDS 2011;25(4):453-462. [http://dx.doi.org/10.1097/QAD.0b013e328341b7f1]

38. Violari A, Cotton MF, Gibb DM, et al. Early antiretroviral therapy and mortality among HIV-infected infants. N Engl J Med 2008;359(21):2233-2244. [http://dx.doi.org/10.1056/NEJMoa0800971]

39. United Nations. Source: UNICEF. Pneumonia and diarrhoea. June 2012. http://www.childinfo.org publications (accessed 20 June 2013). 